\title{
Design of a Centrifugal Blower Adopting Reverse Engineering Approach
}

\author{
Prof. (Dr). M.L Kulkarni ${ }^{1}$, Shubham Goyal ${ }^{2}$, Dhaivat Acharya ${ }^{3}$ \\ Deep Khant ${ }^{4}$, SK Azharuddin ${ }^{5}$ \\ 1, 2, 3, 4, 5 (Department of Mechanical Engineering, MIT-AOE/University of Pune, India)
}

\begin{abstract}
The present project has developed strategy and design procedure for blower which is expected to bring down the lead time during designing through the Reverse Engineering approach. The different dimensions $\&$ geometry of parts of the existing blower were found out by obtaining the Cartesian coordinates of various identified points. Thereafter the required profile and models were developed using this data with the help of CATIA V5 modeler. The Suction condition and other related data's such as "inlet \& outlet diameter", "inlet \& outlet vane angles" \& "vane width" at the inlet and outlet were used to calculate specific data's such as "Absolute velocity of the jet", "velocity at the inlet and outlet", "whirl velocity at outlet" and "exit angle of jet at the vane". The project also covers areas of Geometric Analysis, Fluid Dynamics and Concept of Curve Generation.
\end{abstract}

Keywords: Centrifugal Blower, Design, Reverse Engineering

\section{NOMENCLATURE:}

$\theta$ - Inlet vane angle, degrees

$\phi$ - Outlet vane angle, degrees

$\beta$ - Angle at which jet leaves the vane, degrees

$b_{1}$ - Vane width at inlet, $m$

$\mathrm{b}_{2^{-}}$Vane width at outlet, $\mathrm{m}$

Q- Discharge rate, $\mathrm{m}$

$\mathrm{N}$ - Speed of Impeller, RPM

$\omega$ - Angular Speed of Impeller, $\mathrm{rad} / \mathrm{s}$

R- Base Radius, $m$ $\mathrm{r}_{1}$ - Radius of Impeller at inlet, $\mathrm{m}$

$r_{2}$ - Radius of Impeller at outlet, $m$

$\mathrm{u}_{1}$ - Vane velocity at inlet, $\mathrm{m} / \mathrm{s}$

$\mathrm{u}_{2}$ - Vane velocity at outlet, $\mathrm{m} / \mathrm{s}$

$\mathrm{v}_{1}$ - Absolute velocity of jet, $\mathrm{m} / \mathrm{s}$

$\mathrm{v}_{\mathrm{f} 1}$ - Velocity of flow at inlet, $\mathrm{m} / \mathrm{s}$

$\mathrm{v}_{\mathrm{f} 2}-$ Velocity of flow at outlet, $\mathrm{m} / \mathrm{s}$

$\mathrm{v}_{\mathrm{w} 2}-$ Whirl velocity at outlet, $\mathrm{m} / \mathrm{s}$

\section{Introduction}

A centrifugal blower is a mechanical device for circulating air or other gases. These blowers are used to produce pressurized air at the outlet to the tune of 1.51 bars to 2.75 bars $^{[1]}$. In the following project, Reverse Engineering of backward curved vane blower is used for its subsequent study and research. Reverse Engineering is the process of discovering the technological principles of a device, object, or system through analysis of its structure, function, and operation ${ }^{[2]}$.

In reverse engineering of complex components, ensuring that the new component will be equivalent to and meet or exceed all the specifications of the original design is critical. This technique has become a proven alternative for obtaining replacement parts for existing equipment which may have deteriorated through inservice use or have been damaged by cavitations or its failure in case of Fluid Machinery. Replacing these parts requires experienced design and processes to create a component that will meet the form, fit and function of the original part.

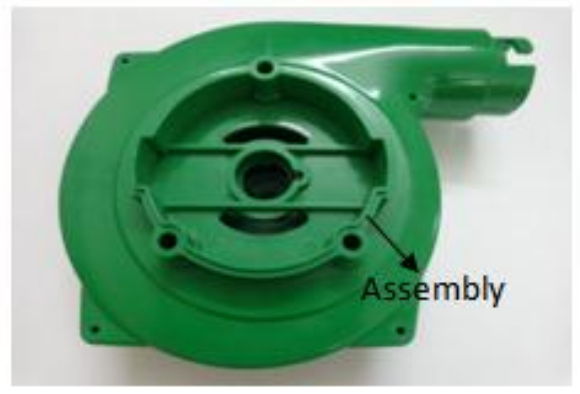

Fig-1 Blower

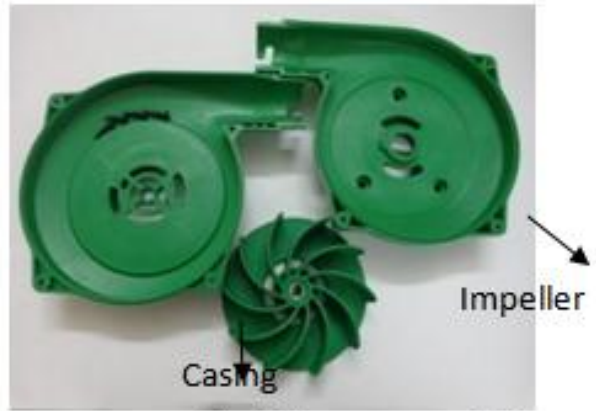

Fig-2 Components of blower 
The above figure illustrates parts of the blower designed. Fluid enters the inlet port at the center of the rotating Impeller, or the suction eye. As the impeller spins in a counter-clockwise direction, it thrusts the fluid outward radially, causing centrifugal acceleration resulting in the creation of vacuum, drawing even more fluid into the inlet. Centrifugal acceleration creates energy proportional to the speed of the impeller ${ }^{[3]}$.

Enhancing the design productivity and incorporating the possible improvements in blower design consumes more time due to the complex geometry of casing and the blades. Attempts are made to address this issue through Reverse Engineering approach. The subsequent study covers the highlighted issues and its solution.

\section{Reverse Engineering}

The following process provides the basic procedure adopted for using the technique of Reverse Engineering in assessing the design of the centrifugal blower. Initially, the backward curved vane blower was dismantled and the parts were individually characterized. Various geometrical tools and techniques were used to carry out the geometrical analysis and the dimensions of the blower were found out. Using various curve generation techniques and mathematical software MATLAB R2007b, we determined the geometrical curves of impeller blade and casing. Subsequently, the calculations were carried out using the mathematical data obtained above to assess the design of the blower. Finally, the simulation was performed from the design of blower which successfully showed the effect on the discharge rate w.r.t the input parameters.

\subsection{Geometrical Analysis of components of blower:}

\subsubsection{Design of Impeller Blade}

In order to deduce nature of the impeller curve, the impeller was placed on a white sheet of paper. A blade at random was chosen and the identified points were marked w.r.t the centre of the hub of impeller using a system of two measuring scales to determine the $\mathrm{x}$ and $\mathrm{y}$ co-ordinates of the selected points. A characteristic curve was generated in MATLAB by plotting the co-ordinates obtained and then a mathematical equation was derived which was then compared to a set of standard mathematical equations. From this comparison the nature of the curve was inferred to be a parabola.

The co-ordinates of the impeller blade and the nature of the curve are as shown below:

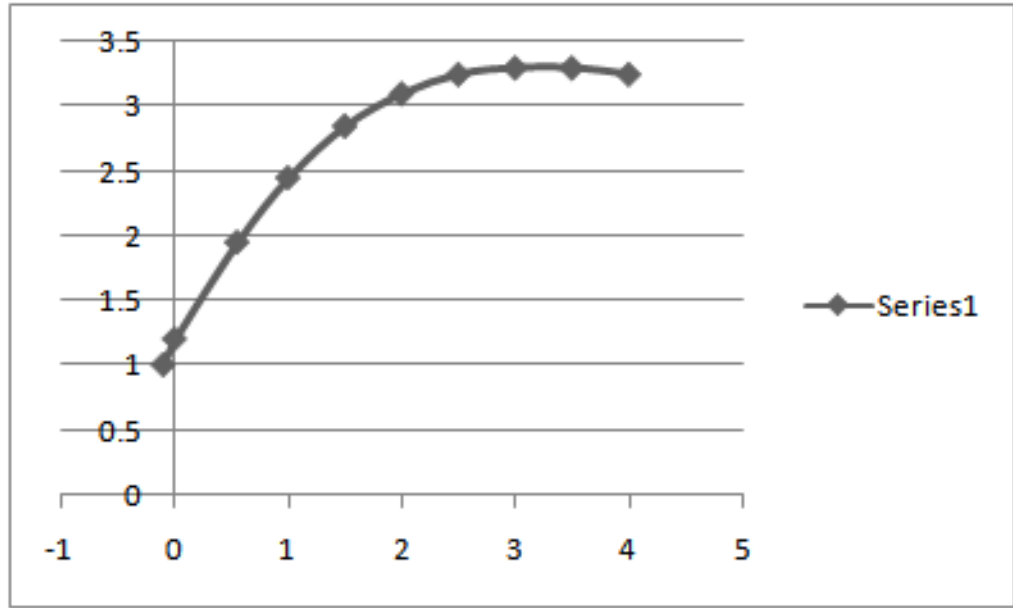

Fig-3 Curve obtained through the identified co-ordinates of impeller

\begin{tabular}{|l|l|}
\hline $\mathrm{X}$ & $\mathrm{Y}$ \\
\hline-0.1 & 1 \\
\hline 0 & 1.2 \\
\hline 0.5 & 1.95 \\
\hline 1 & 2.45 \\
\hline 1.5 & 2.85 \\
\hline 2 & 3.1 \\
\hline 2.5 & 3.25 \\
\hline 3 & 3.3 \\
\hline 3.5 & 3.3 \\
\hline 4 & 3.25 \\
\hline
\end{tabular}

Table-2 Co-ordinates of impeller blade 
The obtained equation is given below:

Where,

$$
(x)=a x^{2}+b x+c
$$

$a=-0.2289$

$b=1.409$

$c=1.205$

Thus, the equation of the curve is:

\subsubsection{Design of Volute Casing}

$$
f(x)=-0.2289 x^{2}+1.409 x+1.205
$$

When the air enters at the suction of the impeller, the shape of the casing provides the necessary pressure to the air ${ }^{[4]}$. The shape of the casing was determined using the arc method from the measured values of inlet radius and the outlet radius of the casing. Thus the nature of the curve so obtained was inferred to be a volute.

Volute base circle radius $=68 * 10^{-3} \mathrm{~m}$

Volute exit radius $=84 * 10^{-3} \mathrm{~m}$

The details of the Arc Method employed are given below:

I. The two points A and B were marked at a distance equal to the value of inlet and outlet radius respectively from the centre point at an angle of $\frac{3 \pi}{2}$.

II. The region points A and B were divided into 6 equal parts as show below.

III. The distance by which the each segment was increased subsequently is

$$
\frac{\left(84 * 10^{-3}\right)-\left(64 * 10^{-3}\right)}{6}=2.667 * 10^{-3} \mathrm{~m}
$$

For eg: $-68 * 10^{-3}+2.667 * 10^{-3}=66.667 * 10^{-3}$

Where, $\quad \mathrm{OA}=68 * 10^{-3} \mathrm{~m}$

$$
\mathrm{OP}=66.667 * 10^{-3} \mathrm{~m}
$$

IV. Similarly all the points were marked. Now the arcs were cut from the centre O using the values of OA \& OP and thus the curve was drawn taking the intersection points of the arc as a centre.

Using this method the entire volute curve was drawn.

The curve obtained is shown below:

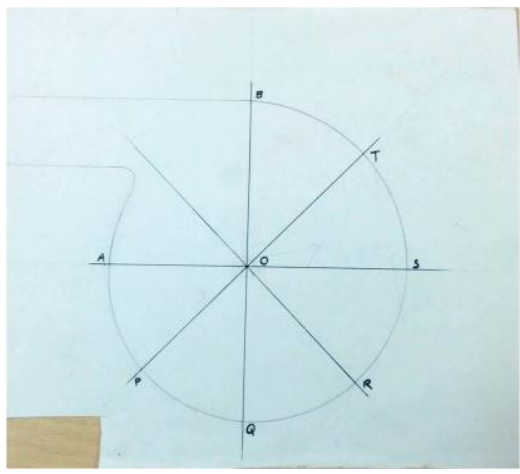

Fig-4 Volute Casing

The magnitude of the measured parameters/ nature of the entities are given in Table-3:

Table-3 Measured Values/Entities using R.E

\begin{tabular}{|c|c|c|}
\hline Serial No. & Particulars & Measured Parameters/Entity \\
\hline 1. & $\theta$ & $40^{\circ}$ \\
\hline 2. & $\phi$ & $50^{\circ}$ \\
\hline 3. & $\mathrm{~N}$ & $13000 \mathrm{rpm}$ \\
\hline 4. & $\mathrm{~b}_{1}$ & $18 \times 10^{-3} \mathrm{~m}$ \\
\hline 5. & $\mathrm{~b}_{2}$ & $26.20 \times 10^{-3} \mathrm{~m}$ \\
\hline 6. & $\mathrm{r}_{1}$ & $10.67 \times 10^{-3} \mathrm{~m}$ \\
\hline 7. & $\mathrm{r}_{2}$ & $55 \times 10^{-3} \mathrm{~m}$ \\
\hline 8. & $\mathrm{R}$ & $68 \times 10^{-3} \mathrm{~m}$ \\
\hline 9. & Blade curve & Parabola \\
\hline 10. & Shape of Casing & Volute \\
\hline
\end{tabular}




\section{Geometrical Modeling}

By using the identified geometry and the dimensions that were found out through the Reverse Engineering approach, the 3-D model for the blower component was developed in CATIA V5. During modeling of various parts of the existing blower, the difficulties faced were as follows:

- Modeling the blade of the impeller

- Surface Modeling of volute casing

As modeling was carried out further, these issues were taken care of by proper inspection and trial and error method. The final assembly model was then prepared by combining all the part models of impellers and volute casing.

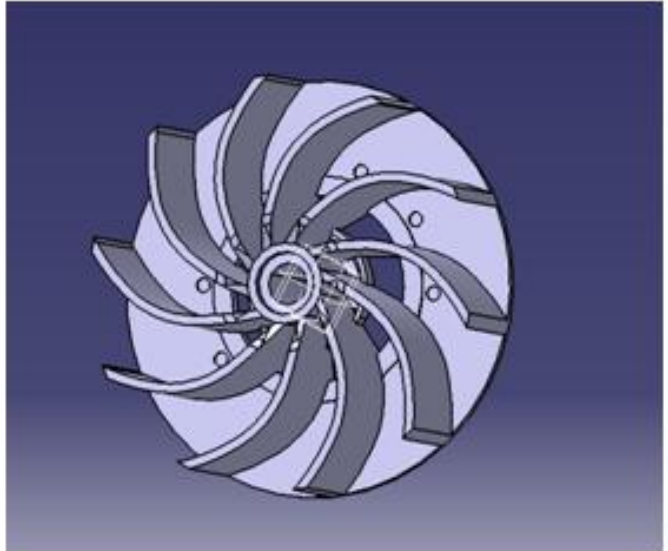

Fig-5 Impeller

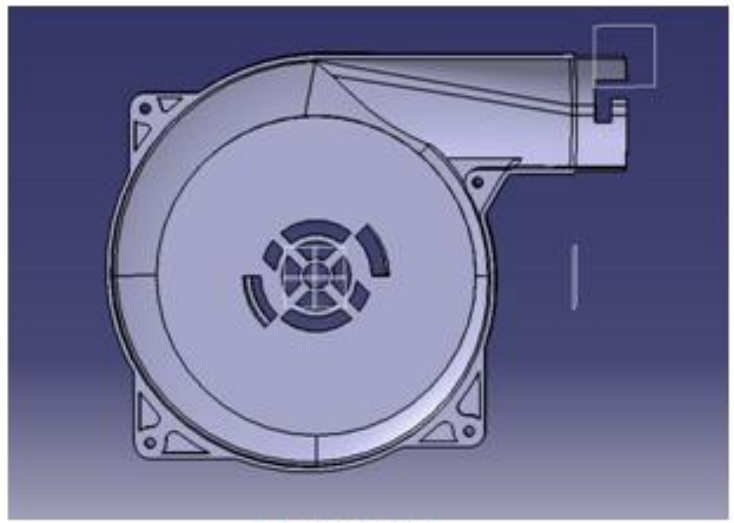

Fig-7 Casing

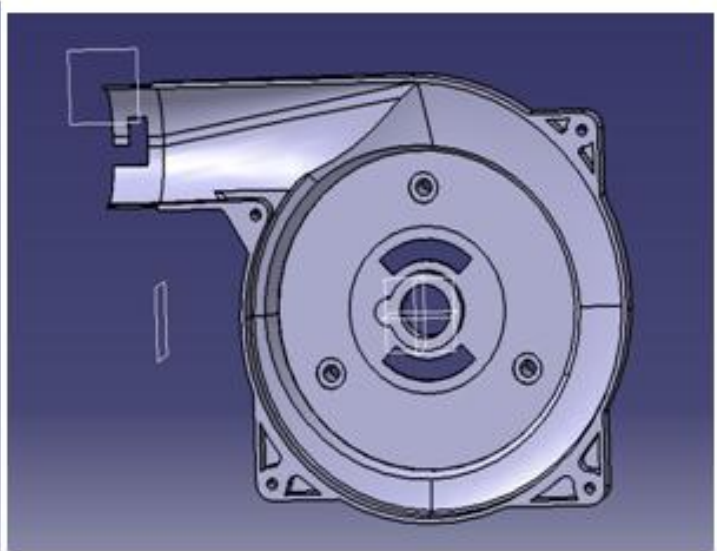

Fig-6 Casing

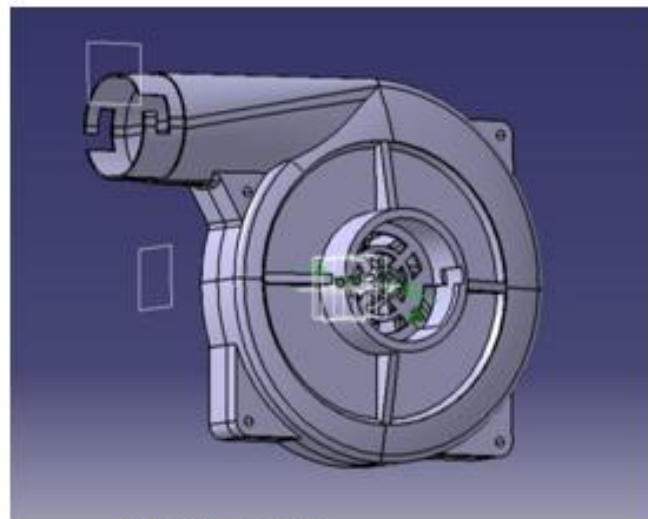

Fig-8 Assembly

IV. Design And Simulation

Forthcoming part provides the design details for the blade and the resulting blower characteristics. The dimensions of the various components obtained through the Reverse Engineering approach are used for the same ${ }^{[5]}$.

$$
\begin{aligned}
& u_{1}=r_{1} * \omega \\
& u_{2}=r_{2} * \omega \\
& \tan \theta=\frac{v_{1}}{u_{1}} \quad \ldots(1) \\
& v_{1}=v_{f_{1}} \quad \ldots(3) \\
& Q=2 \pi r_{1} b_{1} v_{1} \quad \ldots(4) \\
& v_{f_{2}}=\frac{Q}{2 \pi r_{2} b_{2}} \ldots(6)
\end{aligned}
$$




$$
\begin{aligned}
& \tan \phi=\frac{v_{f_{2}}}{u_{2}-v_{w_{2}}}-(7) \\
& \tan \beta=\frac{v_{f_{2}}}{v_{w_{2}}}-\cdots(8)
\end{aligned}
$$

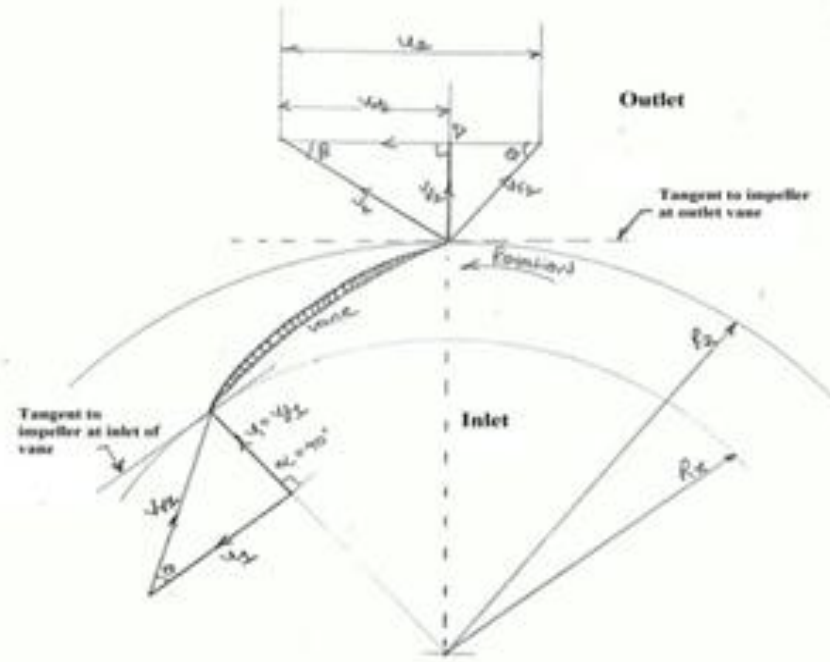

Fig-9 Velocity Triangle

By using the above mentioned equations, the following values were obtained:

- $u_{1}=14.525 \mathrm{~m} / \mathrm{s}$

- $u_{2}=74.87 \mathrm{~m} / \mathrm{s}$

- $v_{1}=12.187 \mathrm{~m} / \mathrm{s}$

- $Q=0.0147 \mathrm{~m}^{3} / \mathrm{s}$

- $v_{f_{2}}=1.623 \mathrm{~m} / \mathrm{s}$

- $v_{w_{2}}=73.507 \mathrm{~m} / \mathrm{s}$

- $\beta=1.264^{\circ}$

\subsection{Simulation}

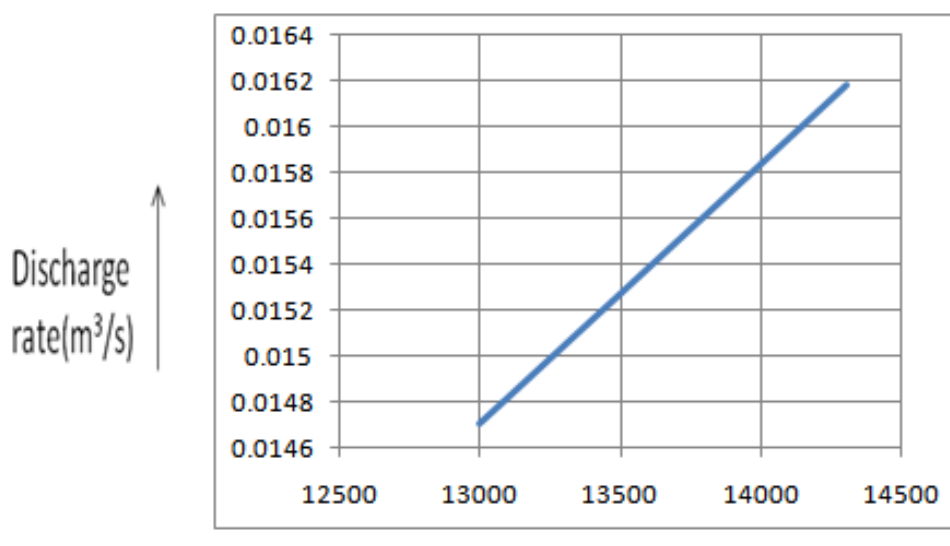

Speed of Impeller (RPM)

Fig-12 Speed of Impeller v/s Discharge rate 

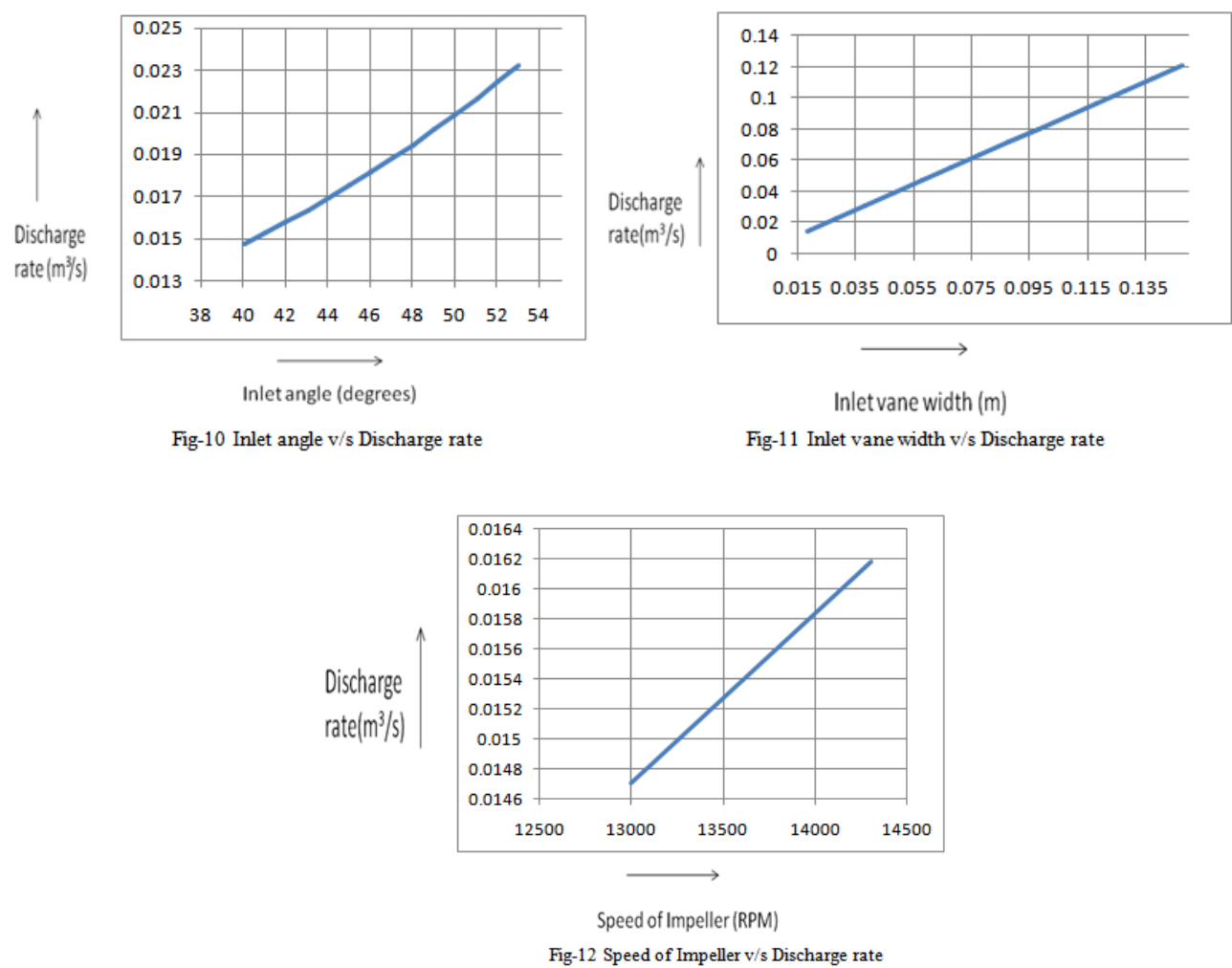

From the above graphs, it follows that:

1) Discharge rate increases with increase in inlet angle- This is because with the increase in inlet angle, the amount of air handled between the two successive blades of the impeller increases which leads to increase of discharge rate.

2) Discharge rate increases with increase in inlet vane width- This is because with the increase in inlet vane width, the area available for air increases. Thus the air-handling capacity of the blower increases resulting into increase of Discharge rate.

3) Discharge rate increases with increase in Speed of impeller- This is because with the increase in speed of impeller, the suction rate of the air increases. Thus the air-handling capacity of the blower increases resulting into increase of Discharge rate.

\section{Validation}

The parameters used in the designing of blower were calculated using the technique of Reverse Engineering and the data thus obtained was compared to the specifications of the blower provided by the manufacturers and were found to be same.

\section{Conclusion}

The performance evaluation of the developed design of blower shows that;

The necessary design of the blower can be designed as per the method adopted in present work. The parametric solid models of the critical components such as impeller and casing are readily available and thus the modeling time for these components is almost zero. Also the Results of the work can be used to select the blade angle/ vane width \& speed so as to obtain the desired discharge and thus the lead time in design can be reduced considerably.

[1] Fans and Blowers, Bureau of Energy Efficiency.

\section{References}

[2] Inbar raz, Introduction to Reverse Engineering, Software Technologies Ltd, December 2011

[3] Fan selection for air handling, Yaskawa Electric America inc AR HV.

[4] Horia Dumitrescu and Vladimir Cardoş, Flow prediction in a blower casing, Institute of Applied Mathematics P.O. Box 1-24, RO707000 , June 2002.

[5] A.T.Oyelami and et al, Analysis of Radial flow Impellers of Different Configurations, Pacific Journal of Science and Technology, May 2012. 